\title{
Complex analysis of the TP53 tumor suppressor in mantle cell and diffuse large B-cell lymphomas
}

\author{
LENKA ZLAMALIKOVA ${ }^{1}$, MOJMIR MOULIS ${ }^{1}$, BARBORA RAVCUKOVA ${ }^{2}$, KVETOSLAVA LISKOVA $^{1}$, \\ JITKA MALCIKOVA ${ }^{3,4}$, DAVID SALEK ${ }^{4}$, JIRI JARKOVSKY ${ }^{5}$, MILUSE SVITAKOVA ${ }^{1}$, \\ RENATA HRABALKOVA $^{1}$, JAN SMARDA ${ }^{6}$ and JANA SMARDOVA ${ }^{1,2}$
}

\begin{abstract}
${ }^{1}$ Department of Pathology, University Hospital, Brno; ${ }^{2}$ Molecular Genetics Laboratory, Centre for Cardiovascular Surgery and Transplantation, Brno; ${ }^{3}$ Central European Institute of Technology (CEITEC), Masaryk University, Brno; ${ }^{4}$ Department of Internal Medicine - Hematology and Oncology, University Hospital, Brno; ${ }^{5}$ Institute of Biostatistics and Analyses, Faculty of Medicine, and ${ }^{6}$ Department of Experimental Biology, Faculty of Science, Masaryk University, Brno, Czech Republic
\end{abstract}

Received May 6, 2017; Accepted July 14, 2017

DOI: $10.3892 /$ or.2017.5891

\begin{abstract}
Mutations and deletions of the tumor suppressor TP53 gene are the most frequent genetic alterations detected in human tumors, though they are rather less frequent in lymphomas. However, acquisition of the TP53 mutation was demonstrated to be one of the characteristic markers in mantle cell lymphoma (MCL) and diffuse large B-cell lymphoma (DLBCL) and prognostic value of the TP53 status has been recognized for these diseases. We present the complex analysis of the TP53 aberrations in 57 cases of MCL and 131 cases of DLBCL. The TP53 status was determined by functional analyses in yeast (FASAY) followed by cDNA and gDNA sequencing. The level of the p53 protein was assessed by immunoblotting and loss of the TP53-specific locus 17p13.3 was detected by FISH. Altogether, we detected 13 TP53 mutations among MCL cases (22.8\%) and 29 TP53 mutations in 26 from 131 DLBCL cases (19.8\%). The ratio of missense TP53 mutations was $76.9 \%$ in MCL and $82.8 \%$ in DLBCL. The frequency of TP53 locus deletion was rather low in both diseases, reaching 9.3\% in MCL and $15.3 \%$ in DLBCL. The presence of TP53 mutation was associated with shorter overall survival (OS) and progression-free survival (PFS) in MCL. Among DLBCL cases, the TP53 mutations shortened both OS and PFS of patients treated with R-CHOP (rituximab, cyclophosphamide, doxorubicin, vincristine and prednisone) and decreased both OS and PFS of patients with secondary DLBCL disease.
\end{abstract}

Correspondence to: Professor Jana Smardova, Department of Pathology, University Hospital, Jihlavska 20, 62500 Brno, Czech Republic

E-mail: janasmarda@seznam.cz

Key words: mantle cell lymphoma, diffuse large B-cell lymphoma, p53 tumor suppressor, FASAY, TP53 mutation

\section{Introduction}

The p53 tumor suppressor mediates an adequate reaction of cells to stress. It functions mainly as a transcription factor responding to various stress stimuli by controlling expression of its target genes. The $p 53$ protein participates in control of cell cycle, DNA repair, apoptosis, senescence, cell metabolism and maintaining of genome integrity $(1,2)$. Mutations and deletions of the TP53 gene are the most frequent genetic alterations detected in human tumors, though they are rather less frequent in lymphomas $(3,4)$.

Recently, the revision of the World Health Organization (WHO) classification of lymphoid neoplasms was published reflecting and summarizing new achievements in the field. The increasing impact of cytogenetic and molecular approaches, including next-generation sequencing (NGS), was emphasized as they incessantly bring new insights and deepen understanding of the lymphoma development, and thus improve diagnosis, prognosis and treatment of this disease. The biologically relevant subgrouping of heterogeneous diffuse large B-cell lymphomas (DLBCL) and appropriate recognition and classification of double/triple-hit lymphomas (DHL/THL) belong to the highlighted diagnostic topics among mature B-cell lymphomas (5). Concurrently, the European Expert Group on NGS-based Diagnostics in Lymphomas (EGNL) summarized the gathered data and divided recurrent mutations in specific lymphomas into five categories having: i) immediate impact on therapy; ii) diagnostic impact; iii) prognostic impact; iv) potential clinical impact in the near future; and v) mutations to be considered for research purposes only. Tumor suppressor TP53 'gets through' a few categories depending on the particular diagnosis. The assessment of its status is essential for clinical decision-making in patients with chronic lymphocytic leukemia (CLL). In addition, the prognostic value of the TP53 status was recognized in splenic marginal zone lymphoma (SMZL), mantle cell lymphoma (MCL) and the subgroup of DLBCL concordantly bearing MYC translocation (6).

The role of p53 in lymphoma has been attributed to its ability to induce apoptosis. Stressed lymphocytes are prone to apoptosis and disruption of the p53-dependent apoptosis 
seems to be essential for the development and progression of lymphoma. However, direct loss of p53 function by mutation of the TP53 gene is not the only way how to accomplish it. The p53 protein level and function itself might be abrogated by several mechanisms. In addition, inactivation of some other proapoptotic genes (e.g. BIM or NOXA) or hyperactivation of antiapoptotic factors (e.g. Bcl-2) can fulfill this role (3). Current genomic technologies allow deep molecular characterization of particular types of lymphoma and their genomic signatures. Recently, the data have been reviewed and summarized for DLBCL, follicular lymphoma (FL), Burkitt lymphoma (BL), mantle cell lymphoma (MCL), marginal zone B-cell lymphoma (MZBL) and primary mediastinal B-cell lymphoma (PMBL). Acquisition of TP53 mutation was demonstrated as one of characteristic markers for DLBCL and MCL, reaching frequency 20 and $25 \%$, respectively, but not for other lymphomas (7). This supports the concept of alternative ways abrogating the p53-dependent apoptosis. We have been studying the TP53 aberrations in MCL and DLBCL for a long time. In our previous study, we analyzed the TP53 status in 33 cases of MCL and 75 cases of DLBCL and assessed its prognostic impact $(8,9)$. Recently, we have enlarged our cohorts and deepened our effort. In the present study, we present the complex analysis of the TP53 status in 57 cases of MCL and 131 cases of DLBCL and reconsider its impact on outcome of the diseases.

\section{Materials and methods}

Clinical material. Fifty-seven patients diagnosed with MCL (44 men and 13 women) and 131 patients diagnosed with DLBCL (76 men and 55 women) were included in the present study. All patients underwent surgical biopsy of the tumor tissue at the University Hospital Brno and were diagnosed by pathologist according to the WHO classification. Median age at MCL diagnosis was 65 years (ranging from 34 to 80 years). DLBCL cohort comprises 105 de novo cases, 26 patients developed DLBCL as a secondary tumor. Median age at DLBCL diagnosis was 57 years (ranging from 20 to 85 years). For all patients, the fresh-frozen tissue samples as well as formalin-fixed, paraffin-embedded (FFPE) tumor tissue blocks were available. All patients were included in this study only after signing the informed consent approved by the ethics committee of the hospital.

FASAY and split assay. FASAY was performed as previously described $(10,11)$ with small modifications. Total RNA was extracted using NucleoSpin RNA kit (New England BioLabs, Inc., Ipswich, MA, USA) and stored at $-80^{\circ} \mathrm{C}$ until further processing. cDNA was synthesized by ProtoScript ${ }^{\circledR}$ II (New England BioLabs) using primer oligo $(\mathrm{dT})_{12}$. PCR was performed using primers P3 (5'-CCT TGC CGT CCC AAG CAA TGG ATG AT-3'), P4 (5'-ACC CTT TTT GGA CTT CAG GTG GCT GGA GT-3') and Phusion DNA polymerase (New England BioLabs). Yeast cells were co-transformed with the PCR product, linearized pSS16 plasmid and salmon sperm DNA carrier (Life Technologies, Inc., Carlsbad, CA, USA) by the lithium acetate procedure (12). Transformed yeast cells were plated on minimal medium lacking leucine and containing adenine $(5 \mu \mathrm{g} / \mathrm{ml})$, followed by incubation at $35^{\circ} \mathrm{C}$ for 2-3 days and then at room temperature for 2-3 days. For split assay, PCR of the TP53 5'- part was performed with primers P3 and P17 (5'-GCC GCC CAT GCA GGA ACT GTT ACA CAT-3'), the 3'- part with primers P4 and P16 (5'-GCG ATG GTC TGG CCC CTC CTC AGC ATC TTA-3'). Yeast cells were transformed with linearized vectors $\mathrm{pFW} 35$ and pFW34 (13).

FASAY deduces the functional status of p53 from color of colonies of transformed yeast cells. Expression of functional p53 results in formation of large white colonies, inactive p53 leads to smaller red ones. The background frequency of red yeast colonies typically does not exceed $10 \%$. Thus, samples providing $<10 \%$ of red colonies are considered to contain only wild-type TP53 alleles, while samples providing $>10 \%$ of red colonies are suspicious to possess a TP53 mutation. The ratio of red colonies scoring between 10 and $20 \%$ can result from a presence of clonal TP53 mutation in rather small fraction of cells or from increased rate of RNA degradation. To distinguish these two possibilities, version of FASAY, called split assay, was established. In the split assay, the 5'- and 3'- parts of the TP53 cDNA are tested separately (13).

Purification of the plasmids from transformed yeast cells and sequencing of the TP53 cDNA. Yeast cells from individual yeast colonies were harvested, resuspended in TSN (2\% Triton X-100, 1\% SDS, $100 \mathrm{mM} \mathrm{NaCl}, 10 \mathrm{mM}$ Tris $\mathrm{pH} 8.0$, $1 \mathrm{mM}$ EDTA), and ground by vortexing with glass beads; plasmid DNA was extracted by phenol/chloroform procedure. The TP53 cDNA was amplified using the $\mathrm{P} 3$ and $\mathrm{P} 4$ primers and Taq polymerase (Life Technologies) and subjected to agarose gel electrophoresis. The PCR product was purified by MinElute PCR purification kit (Qiagen, Hilden, Germany) and sequenced by BigDye Terminator v3.1 cycle sequencing kit (Applied Biosystems, Darmstadt, Germany) using ABI PRISM 3130x Genetic Analyzer (Applied Biosystems).

Isolation and sequencing of the TP53 gDNA. Genomic DNA was isolated from FFPE blocks or from the frozen tumor samples using Puregene DNA Isolation kit (Gentra Systems, Minneapolis, MN, USA) according to the manufacturer's instructions. The TP53 exons were amplified by PCR and automated fluorescent sequencing was performed by using BigDye Terminator kit and ABI 3130 Sequencer (Applied Biosystems, Carlsbad, CA, USA). Either a picked exon of interest or all exons 2-11 were analyzed. Primers and conditions were adopted from IARC TP53 Database (14).

Immunoblotting. Tissue samples were lysed in $150 \mathrm{mM} \mathrm{NaCl}$, $50 \mathrm{mM}$ NaF, $50 \mathrm{mM}$ Tris (pH 8.0), $5 \mathrm{mM}$ EDTA, 1\% NP-40 and $1 \mathrm{mM}$ phenylmethylsulfonyl fluoride in ice for $30 \mathrm{~min}$, and the cell extract was centrifuged at $17,000 \mathrm{x} \mathrm{g}$ for $30 \mathrm{~min}$ to remove cell debris. Protein concentration was measured by the Bradford assay. Solubilized proteins were resolved by $10 \%$ SDS-PAGE and transferred onto a nitrocellulose membrane. Blots were blocked in 0.1\% Tween-20 and 5\% low-fat milk in phosphate-buffered saline (PBS) for $1 \mathrm{~h}$ and probed with anti-p53 mouse monoclonal antibody DO-1 (Dako, Glostrup, Denmark) and PC10 (Abcam, Cambridge, UK) at $4^{\circ} \mathrm{C}$. Blots were developed with Dako peroxidase-conjugated rabbit anti-mouse immunoglobulin (Dako) using the ECL chemi- 
Table I. Summary of the TP53 status analyses in samples of mantle cell lymphoma.

\begin{tabular}{|c|c|c|c|c|c|c|}
\hline Case & FASAY $^{\mathrm{a}}$ & cDNA/gDNA sequencing & TP53 status & $\mathrm{FISH}^{\mathrm{b}}$ & IB & Comment \\
\hline M6 & 88.3 & c. $844 C>T$ & p.R282W & 69.0 & +++ & \\
\hline M9 & 32.2 & c. $712 \mathrm{~T}>\mathrm{A}$ & p.C238S & 28.0 & +++ & \\
\hline M14 & 89.6 & c. $524 \mathrm{G}>\mathrm{A}$ & p.R175H & 0 & +++ & \\
\hline M17 & 82.5 & c. $488 \mathrm{~A}>\mathrm{G}$ & p.Y163C & 6.6 & +++ & \\
\hline M19 & 92.0 & c. $818 \mathrm{G}>\mathrm{A}$ & p.R273H & 0 & ++ & \\
\hline M20 & 90.6 & c. $742 \mathrm{C}>\mathrm{T}$ & p.R248W & 48.0 & +++ & \\
\hline M22 & 75.4 & c. $824 \mathrm{G}>\mathrm{A}$ & p.C275Y & 0 & ++ & \\
\hline M27 & 33.1 & c. $329 \mathrm{G}>\mathrm{T}$ & p.R110L & 0 & ++ & $\mathrm{ts}^{\mathrm{c}}$ \\
\hline M31 & 17.6 & c. 425 delC & p.P142Lfs*27 & 3.4 & - & RNA decay \\
\hline M35 & 60.3 & c.681insT & p.D228* & 0 & ++ & Truncated protein \\
\hline M42 & 75.6 & c. $838 \mathrm{~A}>\mathrm{G}$ & p.R280G & 9.6 & +++ & \\
\hline M43 & 39.9 & c. $991 \mathrm{C}>\mathrm{T}$ & p.Q331* & 8.3 & +++ & Truncated protein \\
\hline M55 & 78.8 & c. $818 \mathrm{G}>\mathrm{A}$ & p.R273H & 61.0 & & \\
\hline
\end{tabular}

IB, immunoblotting; ${ }^{a}$ percentage of red colonies; ${ }^{b}$ percentage of cells with TP53 locus deletion; 'temperature-sensitive mutant.

luminescence detection kit (GE Healthcare UK, Ltd., Little Chalfont, UK).

Fluorescence in situ hybridization (FISH). FISH was performed on tissue sections prepared from FFPE blocks. For the TP53-specific locus analysis, the ZytoLight ${ }^{\circledR}$ SPEC TP53/CEN17 Dual Color Probe were used (ZytoVision GmbH, Bremerhaven, Germany). Hybridization was performed according to the manufacturer's instructions. Images were scanned by DM 5500 B microscope equipped with Leica DFC290 HD camera. Fluorescence signals were analyzed using Leica LAS X software (Leica Microsystems GmbH, Wetzlar, Germany). One hundred cells per case were analyzed. The cut-off level was defined by the mean value plus three times the SD of the frequency of control cells exhibiting one TP53 and two CEN17 signals (9.6\%).

Statistical analyses. Standard descriptive statistics was applied in the analysis; absolute and relative frequencies for categorical variables and median supplemented with minimum-maximum range for continuous variables. The influence of monitored parameters on survival and progressfree survival was assessed by hazard ratio estimates from univariate Cox models. Graphic visualization of patient's survival according to monitored parameters was performed using Kaplan-Meier survival curves. Statistical significance of differences in survival among groups of patients was tested using the log-rank test. Alpha $=0.05$ was used as a level of statistical significance. Analyses were performed in statistical software IBM SPSS Statistics 22.0.0.1 for Windows (IBM Corp., Armonk, NY, USA).

\section{Results}

Assessment of the TP53 status in MCL cases. In our previous study we analyzed the TP53 status in tumor tissues of 33 MCL patients, detecting TP53 mutations in 9 of them.

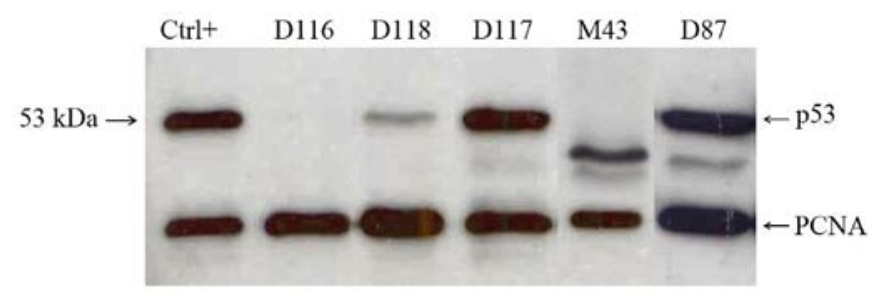

Figure 1. Assessment of the p53 protein level in tumor tissue by immunoblotting using DO-1 antibody. To control for sample loading, the same blot was probed with a PCNA-specific antibody PC10. Ctrl+, positive control: human cell line BT474-bearing the E285K mutation.

Recently, we extended the cohort and enrolled 24 new patients. First, we isolated RNA from frozen tumor tissue, performed FASAY and assessed frequency of red colonies. Twenty cases scored under the $10 \%$ cut-off level, while 4 cases scored clearly above it (ranged from 39.9 to $78.8 \%$ ). For the positive cases, the TP53 expression vector was recovered from 3 to 6 red yeast colonies, and these cDNAs were used as templates for DNA sequencing. In all cases, unambiguous clonal TP53 mutation was identified (Table I). We found that: i) two missense mutations (cases M42 and M55), including the p.R273H that appeared in the cohort for the second time; ii) one nonsense mutation (M43; p.Q331*); and iii) one mutation (M35) representing the frame shift mutation due to one nucleotide insertion and causing formation of premature termination codon (PTC). For the cases M35 and M43, gDNA was isolated from frozen tumor tissue and the corresponding TP53 exons ( 7 and 9, respectively) were amplified by PCR and sequenced. Both mutations were confirmed.

The p53 protein accumulation in tumor tissue of all newly enrolled cases was assessed by immunoblotting using the p53-specific DO-1 monoclonal antibody (Fig. 1). Among 24 cases, 9 exhibited either strong (+++) or intermediate (++) p53 level, including all 4 cases bearing a clonal TP53 mutation. In cases M35 and M43, the truncated p53 proteins were detected corresponding to the shortened coding sequence terminated 
OS

---With TP53 mutation $(\mathrm{N}=13)$

-Without TP53 mutation ( $\mathrm{N}=44)$

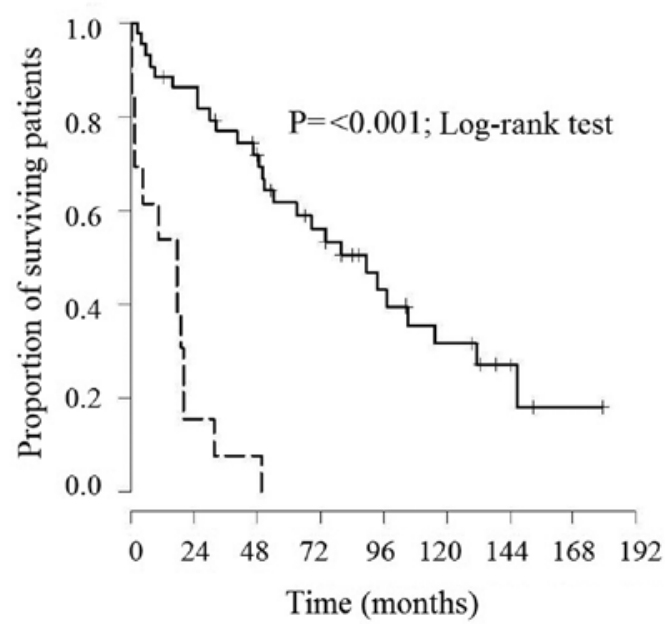

PFS

---With TP53 mutation ( $\mathrm{N}=13)$

- Without TP53 mutation (N=44)

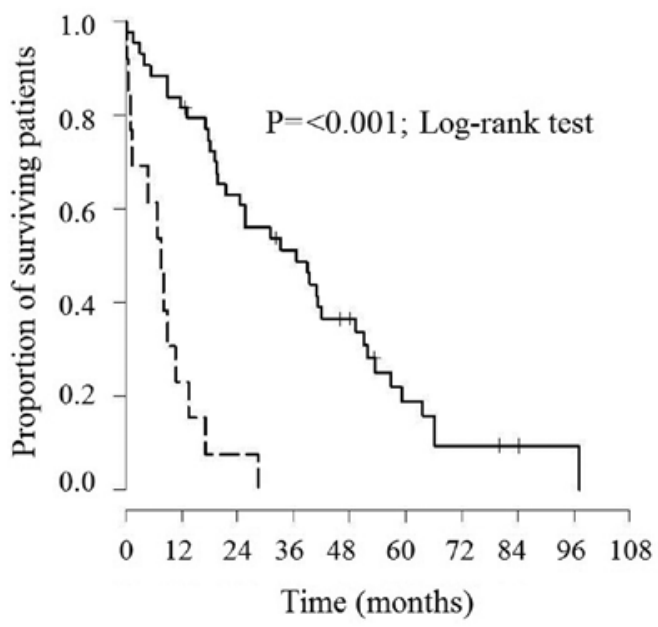

Figure 2. Difference in overall and progression-free survival of MCL patients depending on the presence of the TP53 mutation.

by the PTC causing TP53 mutation. The remaining 15 cases exhibited low (+) or none level (-) of the p53 protein.

We performed FISH analyses of the TP53 alleles using the locus-specific and centromeric probes. We analyzed 22 cases, including all cases bearing the TP53 clonal mutation. Two cases, including M55, scored positive. The other case with the loss of the TP53-specific locus, M47, did not evince a TP53 mutation by FASAY. Therefore, we isolated DNA from the frozen tumor tissue and performed gDNA sequencing of exons 2-11. No TP53 mutation was detected.

Characteristics of TP53 mutations detected in MCL. Altogether, we detected 13 TP53 mutations (Table I) among 57 MCL cases $(22.8 \%)$. All 13 mutations were localized in the region coding for DNA-binding domain. Ten mutations (76.9\%) were the missense ones, including the one that was temperature sensitive. Only one mutation, p.R273H, appeared repeatedly in two cases. There were three mutations causing PTC formation (in positions 169, 228 and 331, respectively); one of them was the nonsense one, one insertion and one deletion. Only the termination codon in position 169 (case M31) induced the mRNA degradation via the PTC-mediated mRNA decay mechanism as we previously showed (8). It was also the only mutation which was not associated with high level of the p53 protein in nuclei of tumor cells. Concerning the TP53 wild-type cases, the p53 protein accumulation was found in 5 of them, 2 cases were not analyzed. Altogether, the p53 protein was accumulated in 17 cases $(30.9 \%)$ and the concordance between the TP53 mutation and the p53 protein accumulation was $89.1 \%(49 / 55)$. The loss of TP53-specific locus was analyzed altogether in 54 MCL cases. Five positive cases were detected (9.3\%); 4 of them bore concurrently missense mutation on the other TP53 allele. Nine TP53 mutation-positive cases were negative for the TP53-specific locus loss (Table I).

Correlation between the TP53 status and MCL outcome. In our previous study, $33 \mathrm{MCL}$ patients were enrolled, female represented $24.2 \%$, and the average age at the time of diag- nosis was 63.4 years. Seventy percent of patients were in stage IV. Their median survival time reached 51.7 months and we showed that TP53 mutation (present in $27.3 \%$ of cases) was associated with shorter overall survival $(\mathrm{P}=0.045)(8)$. The enlarged cohort comprised $57 \mathrm{MCL}$ patients (average age, 65 years; $22.8 \%$ of female; $86 \%$ in stage IV) with median survival time 48.4 months. In this enlarged cohort, we confirmed that the presence of TP53 mutation ( $22.8 \%$ cases) was associated not only with shorter overall survival but also with shorter progression-free survival ( $\mathrm{P}<0.001)$ (Fig. 2).

Assessment of the TP53 status in DLBCL cases. In our previous study we analyzed the TP53 status in tumor tissue of 75 DLBCL patients including 54 de novo cases, detecting 16 TP53 mutations (21.3\%). The recently enlarged cohort comprises 131 patients including 105 de novo cases. First, we isolated RNA from frozen tumor tissue of all 56 new cases, performed FASAY/split assay and assessed the frequency of red colonies. Forty-six cases scored under the $10 \%$ cut-off level and were considered to lack the TP53 mutation. The other 10 cases scored above the cut-off level (ranged from 22.7 to $93.8 \%$ ). The cDNA isolated from the positive yeast colonies was sequenced and 13 TP53 mutations were detected (Table II). Three cases (D86, D87 and D88) bore two mutations, one in each allele. The missense mutations were found in both D97 and D99 cases: one fully inactivating and the second one temperature-sensitive (p. V157A and p. S127T), based on the specific phenotypes of transformed yeast colonies (15). Yet, another temperature-sensitive mutation (p.Y234H) was detected in case D78. In case D87, combination of nonsense mutation in one allele and short insertion/splicing mutation in the second allele was detected. In the 3 cases with two independent mutations, the gDNA was isolated from frozen tumor tissues, the corresponding TP53 exons were amplified by PCR and sequenced; all 6 mutations detected on the cDNA level were clearly confirmed (Table II).

The p53 protein accumulation was assessed by immunoblotting in tumor tissue of all 56 newly enrolled cases 
Table II. Summary of the TP53 status analyses in samples of diffuse large B-cell lymphoma.

\begin{tabular}{|c|c|c|c|c|c|c|}
\hline Case & FASAY $^{a}$ & cDNA/gDNA sequencing & TP53 status & FISH $^{\mathrm{b}}$ & IB & Comment \\
\hline D3 & 70.0 & c. $403 \mathrm{~T}>\mathrm{C}$ & p.C135R & 63.7 & +++ & $\mathrm{ts}^{\mathrm{c}}$ \\
\hline D4 & 94.0 & c.177_182del & p.P60_D61del & 49.1 & $+++^{\mathrm{c}}$ & Truncated protein \\
\hline D5 & 21.7 & c. $730 \mathrm{G}>\mathrm{A}$ & p.G244S & 0.0 & & \\
\hline D7 & 96.8 & c.734G $>\mathrm{A}$ & p.G245D & 0.0 & +++ & \\
\hline D8 & 12.1 & c. $.586 \mathrm{C}>\mathrm{T}$ & p.R196* & 0.0 & - & RNA decay \\
\hline D18 & 28.4 & c. $818 \mathrm{G}>\mathrm{A}$ & p.R273H & 0.0 & + & \\
\hline D28 & 78.0 & $\mathrm{C} 403 \mathrm{~T}>\mathrm{C}$ & p.C135R & 27.5 & + & \\
\hline D30 & 73.6 & c.761T $>A$ & p.I254N & 22.4 & + & $\mathrm{ts}^{\mathrm{c}}$ \\
\hline D35 & 32.0 & c. $770 \mathrm{~T}>\mathrm{G}$ & p.L257R & 18.8 & - & \\
\hline D40 & 60.0 & c. $713 \mathrm{G}>\mathrm{T}$ & p.C238F & 0.0 & +++ & \\
\hline D44 & 83.0 & c. $.815 \mathrm{~T}>\mathrm{G}$ & p.V272G & 46.0 & +++ & \\
\hline D46 & 46.3 & c. $817 \mathrm{C}>\mathrm{T}$ & p.R273C & 0.0 & +++ & \\
\hline D49 & 74.5 & c. $524 \mathrm{G}>\mathrm{A}$ & p.R175H & 29.6 & +++ & \\
\hline D50 & 80.3 & c. $742 \mathrm{C}>\mathrm{G}$ & p.R248G & 0.0 & - & \\
\hline D66 & 62.4 & c.826-835del10 & p.A276Gfs*66 & 1.9 & $+^{\mathrm{c}}$ & Truncated protein \\
\hline D72 & 74.1 & c. $818 \mathrm{G}>\mathrm{A}$ & p.R273H & 1.8 & +++ & \\
\hline D78 & 53.0 & c.700T $>C$ & p.Y234H & 0.4 & ++ & $\mathrm{ts}^{\mathrm{c}}$ \\
\hline D86A & 80.8 & c. $406 \mathrm{C}>\mathrm{G}$ & p.Q136E & 0.0 & +++ & \\
\hline D86B & & c.470T $>C$ & p.V157A & & & $\mathrm{ts}^{\mathrm{c}}$ \\
\hline D87A & 47.9 & $\begin{array}{l}\text { c.783-2A }>\mathrm{T} \\
\left(\mathrm{r} .783 \_784 \text { insTGT }\right)\end{array}$ & p.S261_G262insC & 0.0 & +++ & \\
\hline D87B & & c. $958 \mathrm{~A}>\mathrm{T}$ & p.K320* & & & \\
\hline D88A & 93.8 & c. $376 \mathrm{~T}>\mathrm{G}$ & p.Y126D & 53.0 & +++ & \\
\hline D88B & & c. $379 \mathrm{~T}>\mathrm{A}$ & p.S127T & & & $\mathrm{ts}^{\mathrm{c}}$ \\
\hline D94 & 22.7 & c. $406 \mathrm{C}>\mathrm{G}$ & p.Q136E & 0.0 & ++ & \\
\hline D105 & 37.8 & c. $743 \mathrm{G}>\mathrm{A}$ & p.R248Q & 8.7 & + & \\
\hline D109 & 63.6 & c. $485 \mathrm{~T}>\mathrm{A}$ & p.I162N & 26.0 & +++ & \\
\hline D117 & 78.2 & c. $839 \mathrm{G}>\mathrm{T}$ & p.R280I & 4.0 & +++ & \\
\hline D124 & 78.8 & c. $794 \mathrm{~T}>\mathrm{G}$ & p.L265R & 44.0 & +++ & \\
\hline D131 & 61.0 & c. $818 \mathrm{G}>\mathrm{A}$ & p.R273H & 16.0 & ++ & \\
\hline
\end{tabular}

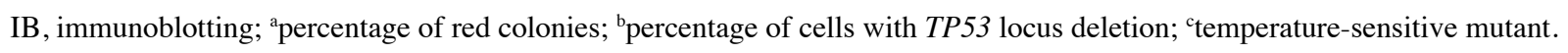

(Fig. 1). Ten cases exhibited strong (+++) p53 protein level, including 6 cases bearing a clonal TP53 mutation. No p53 protein was detected in 4 cases with a clonal missense TP53 mutation. Four cases with no TP53 mutation found by functional analysis scored positive by immunoblotting. Forty-three cases without a TP53 mutation scored negative by immunoblotting.

We performed FISH analysis of the TP53 alleles using the locus-specific and centromeric probes in all 57 newly enrolled cases. Ten cases scored positive, including 4 cases bearing the TP53 clonal mutation. Six cases with the loss of the TP53specific locus did not evince a TP53 mutation by FASAY. We randomly chose 2 of them (D113 and D130), isolated gDNA from frozen tumor tissues, amplified and sequenced exons 4-10. No TP53 mutation was detected.

Characteristics of TP53 mutations detected in DLBCL. Altogether, we detected 29 TP53 mutations in 26 tumor samples (Table II) among 131 DLBCL cases (19.8\%). All mutations were localized in the DNA-binding domain-coding region. Twenty-four mutations $(82.8 \%)$ were the missense ones; including 5 temperature sensitive mutations $(20.8 \%)$. The mutation p.R273H appeared repeatedly in 3 cases (D18, D72 and D131), the mutation p.Q136E was detected twice (D86A and D94). Two mutations were the nonsense ones (D8 and D87B) causing PTC formation in positions 196 and 320. Two mutations were recognized as deletions, one in frame (D4), another causing the reading frame shift and PTC formation in position 345 (D66; 9). The TP53 mutation detected in case D87A was the splicing site mutation (c.783-2A $>$ T) resulting in one amino acid insertion at the protein level (p.S261_G262insC), as documented on mRNA from yeast colonies (r.783_784insTGT). Only the termination codon in position 196 (case D8) induced the mRNA degradation via the PTC-mediated mRNA decay mechanism as we previously shown (9). This mutation, as well as other 11 cases with the TP53 mutation, was not associated with high level of the p53 protein in nuclei of tumor cells. The case D66 bearing short 
OS

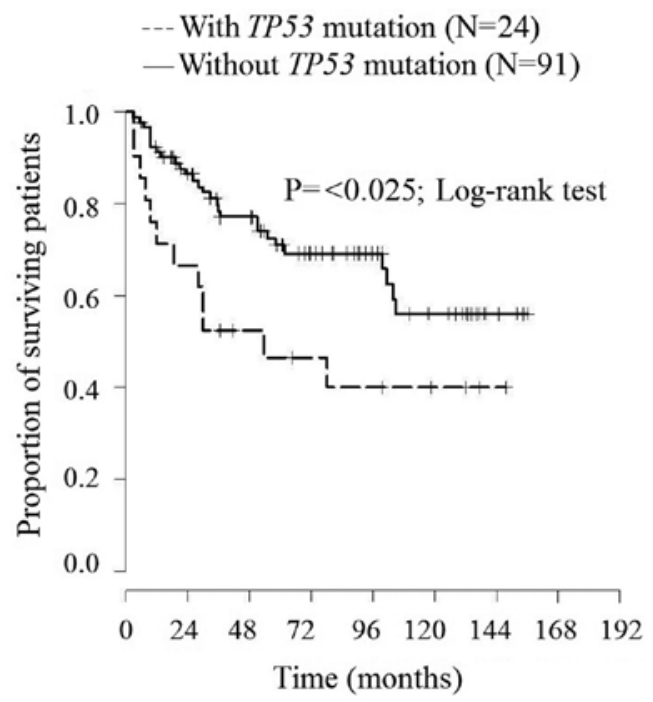

PFS

--- With TP53 mutation $(\mathrm{N}=24)$

- Without TP53 mutation ( $=91)$

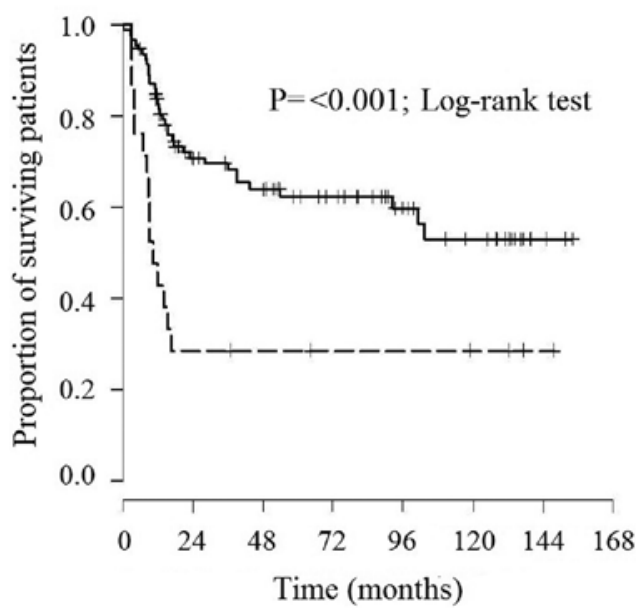

Figure 3. Difference in overall and progression-free survival of DLBCL patients depending on the presence of the TP53 mutation.

deletion and PTC in position 345 was also classified as the p53 protein-negative, although slight $(+)$ band of truncated p53 protein was detected by immunoblotting. The proteins detected in D4 and D87 cases were also truncated (Fig. 1). Concerning the TP53 wild-type cases, the p53 protein accumulation was found in 6 of them. Altogether, the p53 protein was accumulated in 20 cases $(15.3 \%)$ and the concordance between the TP53 mutation and the p53 protein accumulation was $86.3 \%$ (113/131). The loss of TP53-specific locus was analyzed in all 131 DLBCL cases. Twenty positive cases were detected (15.3\%); 10 of them bore concurrently a TP53 mutation. Ten TP53 mutation-positive cases were negative for the TP53specific locus loss (Table II).

Correlation between the TP53 status and DLBCL outcome. In our previous study, 75 DLBCL patients were enrolled. Twenty-one cases developed into DLBCL by transformation from less aggressive disease, 54 patients were de novo cases. Among the de novo DLBCL patients, 46 were treated with R-CHOP therapy. The only statistically significant impact of the TP53 status on clinical parameters was found for the R-CHOP-treated de novo DLBCL patients: the TP53 mutation and/or deletion decreased their progression-free survival $(\mathrm{P}=0.021)$ (9). The enlarged cohort comprised 131 DLBCL patients: 26 secondary and 105 de novo cases. Altogether 115 patients were treated with rituximab. In this extended study, we proved that TP53 mutation shorten both OS and PFS ( $\mathrm{P}=0.029$, $\mathrm{P}=0.001$, respectively) of R-CHOP-treated DLBCL patients and decreases both OS and PFS of patients with secondary DLBCL disease (both $\mathrm{P}=0.021)$ (Fig. 3).

\section{Discussion}

In the present study, we performed detailed analyses of the TP53 status in 24 new cases of MCL and 56 new cases of DLBCL. Extending our previous studies $(8,9)$, we altogether assembled cohorts of $57 \mathrm{MCL}$ and 131 DLBCL cases. This enabled us not only to verify and deepen the previous accom- plishments but also to compare the TP53 status of two different types of lymphoma.

We studied the cohort of MCL patients that was significantly extended, almost doubled, and the results concerning TP53 confirmed the originally reached findings. In addition, the results were in very good agreement with recently published data of other authors. The frequency of the TP53 mutations in the present cohort was $22.8 \%$, compared to $27.3 \%$ in the original one. The frequencies of the TP53 aberrations recently reported by others ranged from 13.7 to $28.0 \%$ depending on cohort size and used methodical approach (16-22). In our cohort, the TP53 mutations occurred more frequently in the aggressive MCL variants (blastoid and pleomorphic) reaching 41.2 and $54.5 \%$, respectively, compared to 14.6 and $13.6 \%$, respectively, in the classical cases. Similar results were reported by Slotta-Huspenina et al (17) who detected the TP53 mutations in $10 \%$ of classical cases and in $91 \%$ of blastoid cases. On the other hand, comparable frequency of the TP53 mutations among classical and blastoid variants (13 vs. $18 \%$ ) was also reported (16). The ratio of missense mutations was $69.2 \%$ in our presented cohort, compared to $88.9 \%$ in the original one. It is comparable to $71.4 \%(14 / 15)$ and $81.8 \%(9 / 11)$ reached by others $(16,19,20)$. This study showed that the presence of TP53 mutation is associated with both shorter overall survival and progression-free survival, while in the original cohort the association between TP53 mutation and PFS did not reach the statistical significance. In some recent studies, the adverse impact of the TP53 mutations and aberrations on OS of MCL patients was confirmed $(16,17)$. We found rather low frequency of the TP53 locus deletion among the MCL cases reaching 9.3 and $9.1 \%$, respectively. This aberration occurs almost exclusively in the cases with the other allele being inactivated by missense mutation. Altogether, only one third (30.8 and 33.3\%, respectively) of our TP53-mutated cases had lost the second TP53 allele. The 17p loss was found in 50\% of TP53 mutated cases and altogether in 32\% of MCL cases by Halldórsdóttir et al (16). Beà et al (18) even found the $17 \mathrm{p}$ alterations in 6 of 8 TP53-mutated cases (75\%) while the one 
of the remaining cases bore two independent TP53 mutations. Delfau-Larue et al (22) found deletion of TP53-specific locus in $22 \%$ of their MCL cases. The proportion of MCL cases with the TP53 locus deletion thus remains rather unclear.

We were unable to find, even in the extended cohort, statistically significant association between the disease course and the p53 protein accumulation or the TP53 locus deletion independently on the presence of TP53 mutation, while the former was documented by Nordström et al (21) and the latter by Delfau-Larue et al (22). In both studies, the size of respective cohorts was substantially larger comprising 127 and 134 cases, respectively. On the other hand, in the large cohort of 119 MCL cases described by Halldorsdóttir et al (16), the presence or absence of TP53 locus, though found in high proportion of cases $(31.9 \%)$ did not influence patient survival, thus confirming similar conclusions made by Stocklein et al (23).

The TP53 status found in our extended DLBCL cohort also confirmed and deepened our original findings and corresponded very well with the data published recently by International DLBCL Rituximab-CHOP Consortium. This crucial, extensive, multicentric study comprises data obtained by analysis of 506 DLBCL patients treated with R-CHOP where 133 TP53 mutations were collected in total (24). In our present cohort, 29 TP53 mutations in 26 tumor samples among 131 DLBCL cases were detected. The total frequency of the TP53 mutations reached $19.8 \%$, compared to $22.1 \%$ documented by the Consortium cohort. The ratio of missense mutations reached $82.8 \%$ in our cohort compared with $81 \%$ described by the Consortium collection. In the Consortium study, the IARC TP53 database (http://www.iarc.fr) was used to retrieve functional level of the detected TP53 mutations. Sixteen mutations were recognized as partially functional, representing $18.2 \%$ (24). Using the functional analysis FASAY for detection of the TP53 mutations allowed us to directly recognize five partially functional, temperature-dependent mutations representing $17.2 \%$ of our TP53 mutations. Collection of TP53 mutations detected by the Consortium was large enough to assemble mutational spectrum. The mutation in codon 273 appearing repeatedly in four our cases was the second most frequent mutation position found by the Consortium (detected in 6 cases). In the most often mutated codon 248 according to the Consortium (mutated in 11 cases), we detected two mutations. In the Consortium study, 17 patients representing $15.2 \%$ of all patients with the mutated TP53 status exhibited multiple mutations. Three of our patients with two independent mutations in separated alleles represented comparable $11.5 \%$ of our cohort of 26 patients with mutated the TP53 gene. From seven Consortium patients carrying a mutation in a splicing site, three bore another TP53 mutation, the missense one. Notably, our only patient bearing the splicing site mutation (c.783-2A $>\mathrm{T}$; p.262insC; D87A) also exhibited the second TP53 mutation (c.958A $>$ T; p.K320*; D87B). Both these mutations are rather rare as the D87A mutation was reported eight times only and D87B twice only according to IARC database (http://www.iarc.fr, version $\mathrm{R} 18 ; 14)$. In our cohort, both these mutations, as well as other multiple mutations, were confirmed by gDNA sequencing.

We found 20 cases with the loss of the TP53 locus, only half of them bearing concurrent TP53 mutation. This rather weak association between TP53 allelic deletion and TP53 mutation was reported also by the Consortium. Similarly to the study, we did not find a significant impact of TP53 allelic deletion on either OS or PFS, thus, supporting conclusion that TP53 mutations and not TP53 locus deletion drive DLBCL progression (24).

In the present study, we showed that the TP53 mutations shorten OS and PFS of DLBCL patients treated with rituximab and decreases both OS and PFS of patients with secondary DLBCL disease. These results significantly extended our previous study (9), where the only statistically significant association was found between the TP53 mutation status and PFS of the R-CHOP-treated de novo DLBCL patients. Nevertheless, the TP53 allelic loss, and the p53 protein accumulation without concerning the presence of the TP53 mutation were not shown to have any clinical impact. It is in good agreement with the Consortium study detecting the TP53 allelic loss in comparable low proportion of cases (12.3\%) and without significant association with decreased OS or PFS (24). In the same study, the p53 protein overexpression analysis by immunohistochemistry was found to have only limited prognostic impact strongly dependent on used cut-offs (24).

The present study allowed us to compare results of the TP53 status analyses of two different lymphoma types, MCL and DLBCL. In many regards, the results were similar. For example, the frequency of TP53 mutations was comparable, reaching $22.8 \%$ in MCL and $19.8 \%$ in DLBCL. These results match well with the recently published comprehensive data presenting 25\% for MCL and $20 \%$ for DLBCL (7). Similarly, the ratio of missense TP53 mutations was comparable for MCL and DLBCL reaching 76.9 and $82.8 \%$, respectively. The detected frequency of TP53 locus deletion was similarly low in both diseases reaching 9.3\% in MCL and 15.3\% in DLBCL. Its low association with TP53 mutations was found also in both diseases. All these results strongly support involvement of the TP53 tumor suppressor mutations, rather than deletion or overexpression, in development and progression of both MCL and DLBCL. Although the frequency of TP53 mutations was very similar in both types of lymphoma, the indispensable sizes of the cohorts allowing conclusive assessment of their clinical impacts are very different due to strong molecular difference between MCL a DLBCL. While MCL represents rather clearly defined type of lymphoma that is easy distinguishable from other lymphomas, DLBCL as diagnostic unit is characterized by its heterogeneity $(5,7,25)$. Any conclusive results would always be conditioned by assembling extensive cohorts of patients.

\section{Acknowledgements}

The present study was supported by the grant NT/137844/2012 of the Internal Grant Agency of the Ministry of Health of the Czech Republic, MH CZ-DRO (FNBr, 65269705), and MEYS CR CEITEC 2020 (LQ1601).

\section{References}

1. Harris SL and Levine AJ: The p53 pathway: Positive and negative feedback loops. Oncogene 24: 2899-2908, 2005.

2. Sherr CJ: Principles of tumor suppression. Cell 116: 235-246, 2004. 
3. Xu-Monette ZY, Medeiros LJ, Li Y, Orlowski RZ, Andreeff M, Bueso-Ramos CE, Greiner TC, McDonnell TJ and Young KH: Dysfunction of the TP53 tumor suppressor gene in lymphoid malignancies. Blood 119: 3668-3683, 2012.

4. Leroy B, Anderson M and Soussi T: TP53 mutations in human cancer: Database reassessment and prospects for the next decade. Hum Mutat 35: 672-688, 2014.

5. Swerdlow SH, Campo E, Pileri SA, Harris NL, Stein H, Siebert R, Advani R, Ghielmini M, Salles GA, Zelenetz AD, et al: The 2016 revision of the World Health Organization classification of lymphoid neoplasms. Blood 127: 2375-2390, 2016.

6. Rosenquist R, Rosenwald A, Du MQ, Gaidano G, Groenen P, Wotherspoon A, Ghia P, Gaulard P, Campo E and Stamatopoulos K; European Research Initiative on CLL (ERIC) and the European Association for Haematopathology (EAHP): Clinical impact of recurrently mutated genes on lymphoma diagnostics: State-of-the-art and beyond. Haematologica 101: $1002-1009,2016$

7. Iqbal J, Naushad H, Bi C, Yu J, Bouska A, Rohr J, Chao W, Fu K, Chan WC and Vose JM: Genomic signatures in B-cell lymphoma: How can these improve precision in diagnosis and inform prognosis? Blood Rev 30: 73-88, 2016.

8. Stefancikova L, Moulis M, Fabian P, Ravcukova B, Vasova I, Muzik J, Malcikova J, Falkova I, Slovackova J and Smardova J: Loss of the p53 tumor suppressor activity is associated with negative prognosis of mantle cell lymphoma. Int J Oncol 36 : 699-706, 2010

9. StefancikovaL,Moulis M,Fabian P, Vasova I,ZedekF,RavcukovaB, Muzik J, Kuglik P, Vranova V, Falkova I, et al: Prognostic impact of p53 aberrations for R-CHOP-treated patients with diffuse large B-cell lymphoma. Int J Oncol 39: 1413-1420, 2011.

10. Flaman JM, Frebourg T, Moreau V, Charbonnier F, Martin C, Chappuis P, Sappino AP, Limacher IM, Bron L and Benhattar J: A simple p53 functional assay for screening cell lines, blood, and tumors. Proc Natl Acad Sci USA 92: 3963-3967, 1995.

11. Smardová J, Nemajerová A, Trbusek M, Vagunda V and Kovarík J: Rare somatic p53 mutation identified in breast cancer: A case report. Tumour Biol 22: 59-66, 2001.

12. Ishioka C, Frebourg T, Yan YX, Vidal M, Friend SH, Schmidt S and Iggo R: Screening patients for heterozygous p53 mutations using a functional assay in yeast. Nat Genet 5: 124-129, 1993

13. Waridel F, Estreicher A, Bron L, Flaman JM, Fontolliet C, Monnier P, Frebourg T and Iggo R: Field cancerisation and polyclonal p53 mutation in the upper aero-digestive tract. Oncogene 14: 163-169, 1997

14. Bouaoun L, Sonkin D, Ardin M, Hollstein M, Byrnes G, Zavadil J and Olivier M: TP53 variations in human cancers: New lessons from the IARC TP53 database and genomics Data. Hum Mutat 37: 865-876, 2016.

15. Grochova D, Vankova J, Damborsky J, Ravcukova B, Smarda J, Vojtesek B and Smardova J: Analysis of transactivation capability and conformation of p53 temperature-dependent mutants and their reactivation by amifostine in yeast. Oncogene 27: 1243-1252, 2008
16. Halldórsdóttir AM, Lundin A, Murray F, Mansouri L, Knuutila S, Sundström C, Laurell A, Ehrencrona H, Sander B and Rosenquist R: Impact of TP53 mutation and 17p deletion in mantle cell lymphoma. Leukemia 25: 1904-1908, 2011.

17. Slotta-Huspenina J,Koch I, de Leval L, Keller G, Klier M, Bink K, Kremer M, Raffeld M, Fend F and Quintanilla-Martinez L: The impact of cyclin D1 mRNA isoforms, morphology and p53 in mantle cell lymphoma: p53 alterations and blastoid morphology are strong predictors of a high proliferation index. Haematologica 97: 1422-1430, 2012.

18. Beà S, Valdés-Mas R, Navarro A, Salaverria I, Martín-Garcia D, Jares P, Giné E, Pinyol M, Royo C, Nadeu F, et al: Landscape of somatic mutations and clonal evolution in mantle cell lymphoma. Proc Natl Acad Sci USA 110: 18250-18255, 2013.

19. Meissner B, Kridel R, Lim RS, Rogic S, Tse K, Scott DW, Moore R, Mungall AJ, Marra MA, Connors JM, et al: The E3 ubiquitin ligase UBR5 is recurrently mutated in mantle cell lymphoma. Blood 121: 3161-3164, 2013

20. Zhang J, Jima D, Moffitt AB, Liu Q, Czader M, Hsi ED, Fedoriw Y, Dunphy CH, Richards KL, Gill JI, et al: The genomic landscape of mantle cell lymphoma is related to the epigenetically determined chromatin state of normal B cells. Blood 123: 2988-2996, 2014.

21. Nordström L, Sernbo S, Eden P, Grønbaek K, Kolstad A, Räty R, Karjalainen ML, Geisler C, Ralfkiaer E, Sundström C, et al: SOX11 and TP53 add prognostic information to MIPI in a homogenously treated cohort of mantle cell lymphoma: a Nordic Lymphoma Group study. Br J Haematol 166: 98-108, 2014.

22. Delfau-Larue MH, Klapper W, Berger F, Jardin F, Briere J, Salles G, Casasnovas O, Feugier P, Haioun C, Ribrag V, et al; European Mantle Cell Lymphoma Network: High-dose cytarabine does not overcome the adverse prognostic value of CDKN2A and TP53 deletions in mantle cell lymphoma. Blood 126: 604-611, 2015.

23. Stöcklein H, Hutter G, Kalla J, Hartmann E, Zimmermann Y, Katzenberger T, Adam P, Leich E, Höller S, MüllerHermelink HK, et al: Genomic deletion and promoter methylation status of Hypermethylated in Cancer 1 (HIC1) in mantle cell lymphoma. J Hematop 1: 85-95, 2008

24. Xu-Monette ZY, Wu L, Visco C, Tai YC, Tzankov A, Liu WM Montes-Moreno S, Dybkaer K, Chiu A, Orazi A, et al: Mutational profile and prognostic significance of TP53 in diffuse large B-cell lymphoma patients treated with R-CHOP: Report from an International DLBCL Rituximab-CHOP Consortium Program Study. Blood 120: 3986-3996, 2012.

25. Lu TX, Young KH, Xu W and Li JY: TP53 dysfunction in diffuse large B-cell lymphoma. Crit Rev Oncol Hematol 97: 47-55, 2016. 